\title{
BMJ Open Efficacy and safety of faecal microbiota transplantation in patients with psoriatic arthritis: protocol for a 6-month, double-blind, randomised, placebo-controlled trial
}

\author{
Maja Skov Kragsnaes, ${ }^{1,2}$ Jens Kjeldsen, ${ }^{3}$ Hans Christian Horn, ${ }^{1}$ \\ Heidi Lausten Munk, ${ }^{1}$ Finn Moeller Pedersen, ${ }^{3}$ Hanne Marie Holt, ${ }^{4}$ \\ Jens Kristian Pedersen, ${ }^{1}$ Dorte Kinggaard Holm, ${ }^{5}$ Henning Glerup, ${ }^{6}$ \\ Vibeke Andersen, ${ }^{7,8}$ Ulrich Fredberg, ${ }^{6}$ Karsten Kristiansen, ${ }^{9,10}$ Robin Christensen, ${ }^{11}$ \\ Torkell Ellingsen ${ }^{1}$
}

To cite: Kragsnaes MS, Kjeldsen J, Horn HC, et al. Efficacy and safety of faecal microbiota transplantation in patients with psoriatic arthritis: protocol for a 6-month, doubleblind, randomised, placebocontrolled trial. BMJ Open 2018;8:e19231. doi:10.1136/ bmjopen-2017-019231

- Prepublication history for this paper is available online. To view these files, please visit the journal online (http://dx.doi. org/10.1136/bmjopen-2017019231).

Received 29 August 2017 Revised 13 February 2018 Accepted 28 February 2018
Check for updates

For numbered affiliations see end of article.

Correspondence to Maja Skov Kragsnaes; maja.kragsnaes@dadlnet.dk

\section{ABSTRACT}

Introduction An unbalanced intestinal microbiota may mediate activation of the inflammatory pathways seen in psoriatic arthritis (PsA). A randomised, placebocontrolled trial of faecal microbiota transplantation (FMT) infused into the small intestine of patients with PsA with active peripheral disease who are non-responsive to methotrexate (MTX) treatment will be conducted. The objective is to explore clinical aspects associated with FMT performed in patients with PSA.

Methods and analysis This trial is a randomised, two-centre stratified, double-blind (patient, care provider and outcome assessor), placebo-controlled, parallel-group study. Eighty patients will be included and randomised (1:1) to either placebo (saline) or FMT provided from an anonymous healthy donor. Throughout the study, both groups will continue the weekly selfadministered subcutaneous MTX treatment, remaining on the preinclusion dosage (15-25 mg/week). The clinical measures of psoriasis and PsA disease activity used include the Short (2-page) Health Assessment Questionnaire, the Dermatology Quality of Life Index, the Spondyloarthritis Research Consortium of Canada Enthesitis Index, the Psoriasis Area Severity Index, a dactylitis digit count, a swollen/tender joint count (66/68), plasma $\mathrm{C}$ reactive protein as well as visual analogue scales for pain, fatigue and patient and physician global assessments. The primary end point is the proportion of patients who experience treatment failure during the 6-month trial period. The number of adverse events will be registered throughout the study.

Ethics and dissemination This is a proof-of-concept clinical trial and will be performed in agreement with Good Clinical Practice standards. Approvals have been obtained from the local Ethics Committee (DK-S-20150080) and the Danish Data Protection Agency (15/41684). The study has commenced in May 2017. Dissemination will be through presentations at national and international conferences and through publications in international peer-reviewed journal(s).
Strengths and limitations of this study

This is a double-blind, randomised, placebo-controlled trial.

- Subcutaneously administered methotrexate treatment.

- The primary end point is based on shared decision-making between patient and physician.

- No feasibility data regarding faecal microbiota transplantation in patients with rheumatic diseases were available when the trial was designed.

- A limitation of the study is that the content of the faecal transplant suspension cannot be fully standardised.

Trial registration number NCT03058900; Pre-results.

\section{INTRODUCTION}

Emerging data suggest a causal relationship between the intestinal microbiota and spondyloarthritis (SpA), thus linking dysbiosis of the complex microbial communities with SpA pathogenesis. ${ }^{1-5}$ Psoriatic arthritis (PsA) is one of five SpA categories in adults which also include ankylosing spondylitis, undifferentiated arthritis, reactive arthritis and arthritis associated with inflammatory bowel disease (IBD). While the association between the gut and the latter two disorders is well established, ${ }^{6}$ only very recently, studies evaluating the faecal microbiota and the presence of subclinical gut inflammation in patients with PsA have coupled this disease to a perturbation of the intestinal microbiota composition. ${ }^{7-12}$

PsA is a distinct, multifaceted inflammatory disease with a diverse clinical spectrum 
and a varied disease course. ${ }^{13}$ The clinical manifestations include peripheral arthritis, enthesitis and/or spondylitis combined with more or less severe psoriatic skin involvement, nail psoriasis and dactylitis. ${ }^{14}$ Nearly half of the patients with both early and established PsA also present with extramusculoskeletal manifestations, which can include bowel (16\%), ocular, cardiovascular or urogenital involvement. ${ }^{15}$ Without disease-modifying intervention, $40 \%-60 \%$ of patients with PsA will develop erosive and deforming joint damage within a few years of disease onset. ${ }^{16}$ Methotrexate (MTX) has long been the preferred conventional synthetic disease-modifying antirheumatic drug (csDMARD) for initial therapy. ${ }^{17}$ However, the evidence for MTX in PsA is poor, and a substantial number of patients does not benefit from such treatment. ${ }^{18}$ Currently, other treatment options may include biological agents such as tumour necrosis factor (TNF- $\alpha$ ) inhibitors aiming to block some of the downstream molecular pathways driving the disease. ${ }^{19}$ Still, these drugs do not target the cause of PsA, which is believed to be multifactorial comprising genetic, immunological and environmental factors. ${ }^{20}$ The interplay between these complex aetiological factors has yet to be fully understood. ${ }^{21} 22$

The classic pathophysiological concept of PsA is that it is an autoimmune disease of the skin and joints and that the pathological processes at both sites are driven by inflammatory responses involving the innate immune system, natural killer cells, T cells and the expression of proinflammatory cytokines, including TNF- $\alpha$, interleukin (IL)-1, interferon- $\gamma$, IL-6, IL-12, IL-15, IL-18 and the IL-17/IL-23 axis. ${ }^{23-27}$ However, although microbial agents including dormant bacteria, mycobacteria, bacterial products and viral antigens have been implicated as potential initiators, ${ }^{28}{ }^{29}$ the true pathophysiological factors triggering the dysregulated immunological cascade underlying the disease remain to be identified.

Intriguingly, it has recently been suggested that mucosal sites exposed to a high load of bacterial antigens, in particular the gastrointestinal tract, may represent the initial site of immunological tolerance break in PsA ${ }^{30}$ Indeed, under normal conditions the host and the microbiota live in harmony and benefit from their mutualistic relationship. However, alterations of the normal intestinal microbiota can affect mucosal immunity which, in turn, can induce local inflammation and elicit systemic effects at distant sites. ${ }^{31}$ Mechanisms through which the intestinal microbiota may be involved in the pathogenesis of PsA include an abnormal activation of the gut-associated lymphoid tissue ${ }^{32}$ a decrease in regulatory T-cell activity $^{33}$ and/or an altered mucosal permeability thus compromising the capacity of the intestine to provide adequate containment of luminal microorganisms and molecules. ${ }^{34} 35$ In support of these theories, several studies have documented subclinical gut inflammation in patients with PsA. ${ }^{36-41}$ Moreover, a recent study reported that several intestinal bacteria including Akkermansia and Ruminococcus were practically absent in patients with PsA.
These commensal bacteria are, in fact, known to play an important role in maintaining gut homeostasis. ${ }^{42}$

\section{Rationale}

If the gut microbiota is the initiator and/or mediator of the common inflammatory pathways seen in PsA, ${ }^{8}$ modifying the intestinal microbiota could be a novel treatment strategy for this disease. ${ }^{1-3} 43$ Faecal microbiota transplantation (FMT) is currently being used to restore the balance of the intestinal microbiota. ${ }^{44}$ Particularly, this procedure has demonstrated $>90 \%$ clinical resolution of recurrent or refractory Clostridium difficile infections. ${ }^{46-50}$ Also, multiple FMTs seem to be able to induce remission in patients with IBD. ${ }^{51}$ Due to these results, FMT is now being tested as a potential novel treatment for other gastrointestinal and extraintestinal diseases. ${ }^{52}$ To the best of our knowledge, no study has yet ascertained the efficacy and safety of FMT in patients with inflammatory rheumatic diseases.

\section{Evidence-based research}

To avoid waste of research, no new studies should be initiated without a systematic review of the existing evidence. ${ }^{53}$ We performed a pragmatic search in the biomedical literature via Pubmed combining different related $\mathrm{MeSH}$ terms: ('Microbiota' (Mesh) OR 'Fecal Microbiota Transplantation'(Mesh) OR 'Faecal Microbiota Transplantation'(Mesh) OR 'Gastrointestinal Microbiome'(Mesh)) AND (arthritis(tiab) OR 'Arthritis' (Mesh) OR 'Arthritis, Psoriatic'(Mesh) OR 'Arthritis, Reactive'(Mesh) OR 'Spondylarthritis'(Mesh) OR 'Arthritis, Gouty'(Mesh) OR 'Arthritis, Rheumatoid'(Mesh) OR 'Psoriasis'(Mesh)). From the search revealing 122 citations, it became clear that the majority of papers were reviews/ editorials $(74(61 \%))$, where the overall conclusion was that the main challenges are to uncover the cause-effect relationship between the intestinal microbiota and rheumatic diseases, and to investigate the potential of microbiome-targeting strategies. ${ }^{13} 5620324354-60$ Also from the published literature it became evident that to date only nine clinical interventional studies trying to modify the intestinal microbiota in patients with arthritis have been performed: one study in patients with $\operatorname{SpA}(\mathrm{n}=63),{ }^{61}$ and one study in enthesis-related arthritis $(n=8)$ reported no beneficial effects of probiotic therapy ${ }^{62}$ whereas one study in juvenile idiopathic arthritis testing exclusive enteral nutrition administration $(n=7)$ found a moderate anti-inflammatory effect on active joints. ${ }^{63}$ Five placebo-controlled trials of probiotic therapy in patients with rheumatoid arthritis ${ }^{64-68}$ (sample size between 26 and 60 patients) reported mixed results. ${ }^{69}$ However, two of these studies demonstrated positive clinical effects of probiotic therapy, which included improvement in Health Assessment Questionnaire (HAQ)-Didability Index pain scale ${ }^{65}$ improvement in the Disease Activity Score of 28 joints and improvement on the $\mathrm{C}$ reactive protein (CRP) concentrations. ${ }^{66}$ No clinical trials performing FMT on patients with arthritis were identified. 


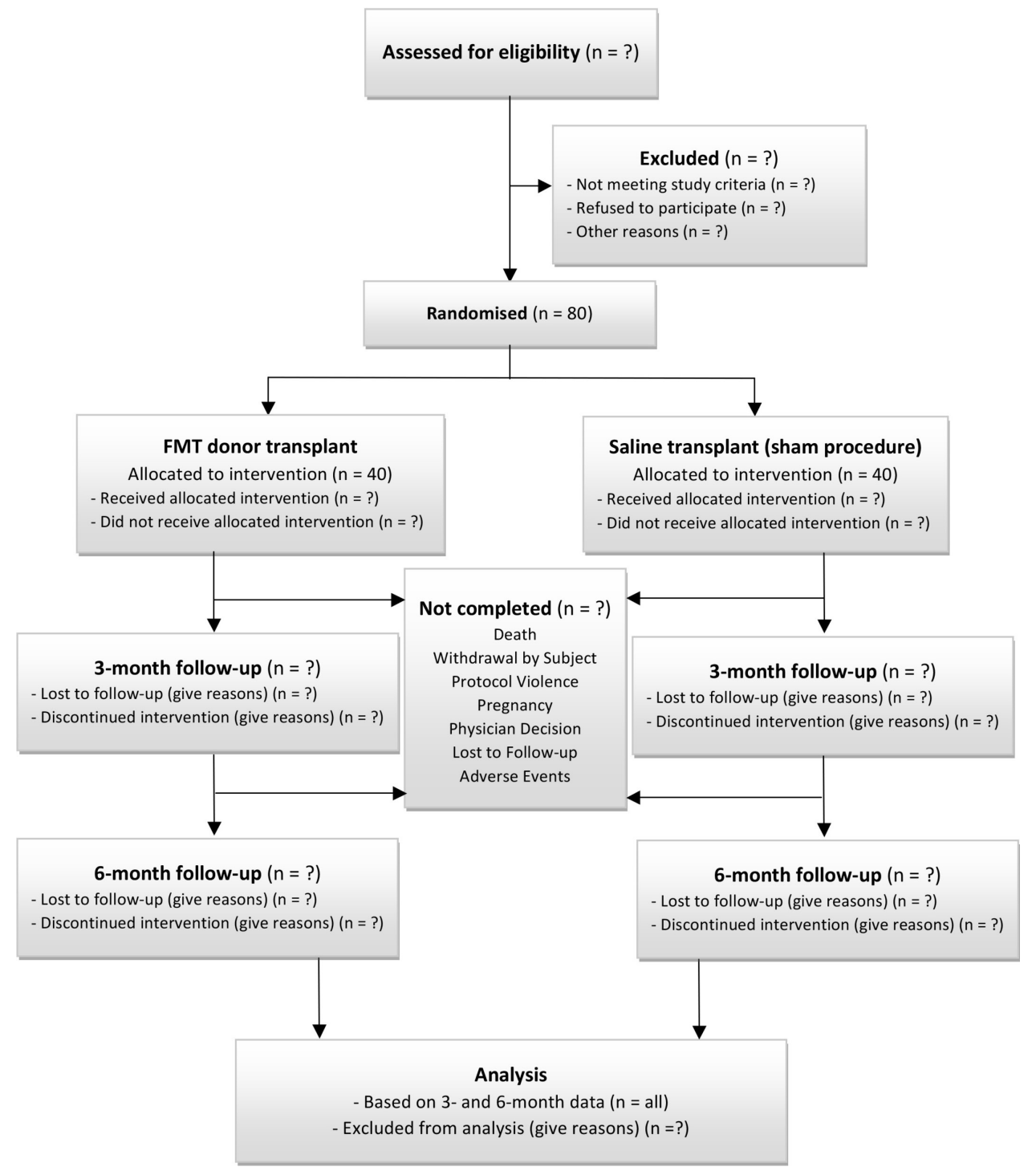

Figure 1 Flow diagram of the randomised, placebo-controlled trial. FMT, faecal microbiota transplantation.

\section{Objective}

The objective of this randomised trial is to explore whether FMT is more effective than placebo in reducing disease activity in patients with PsA with active peripheral arthritis concomitantly treated with weekly subcutaneously administered MTX. In addition, extensive bacterial taxonomic and metagenomic analyses will be performed on faecal samples before and after the FMT to get an indication of the functional capacity of the intestinal microbiota.

\section{METHODS AND ANALYSIS \\ Trial design}

This is a randomised, patient, physician and outcome-assessor blinded, placebo-controlled, 6-month trial, which will be followed by an open-label extension period for a minimum of 2 years. Patients will be randomly assigned in a 1:1 ratio to receive FMT or placebo (sham procedure). Outcome assessment will be based on follow-up by a rheumatologist and is scheduled to occur after 3 and 6 months (with the latter being the primary end point evaluation) (see figure 1 and figure 2).

\section{Participants}

Recruitment will take place at Danish rheumatology outpatient clinics, and patients fulfilling the eligibility criteria will be offered participation. No treatment with biologics within 6 months, and no systemic and/ or local intra-articular or peritendinous steroid injections, or non-MTX csDMARD treatment, or antibiotics are allowed within 3 months prior to inclusion. Non-steroidal anti-inflammatory drugs (NSAIDs) must be paused within 14 days of study inclusion. Patients, who do not wish to participate, will be characterised by sex and age. The recruitment has commenced in May 2017 and will continue until 2019.

\section{Patients with psoriatic arthritis}

A total of 80 patients with PsA will be enrolled, and they will have to meet the following eligibility criteria: 


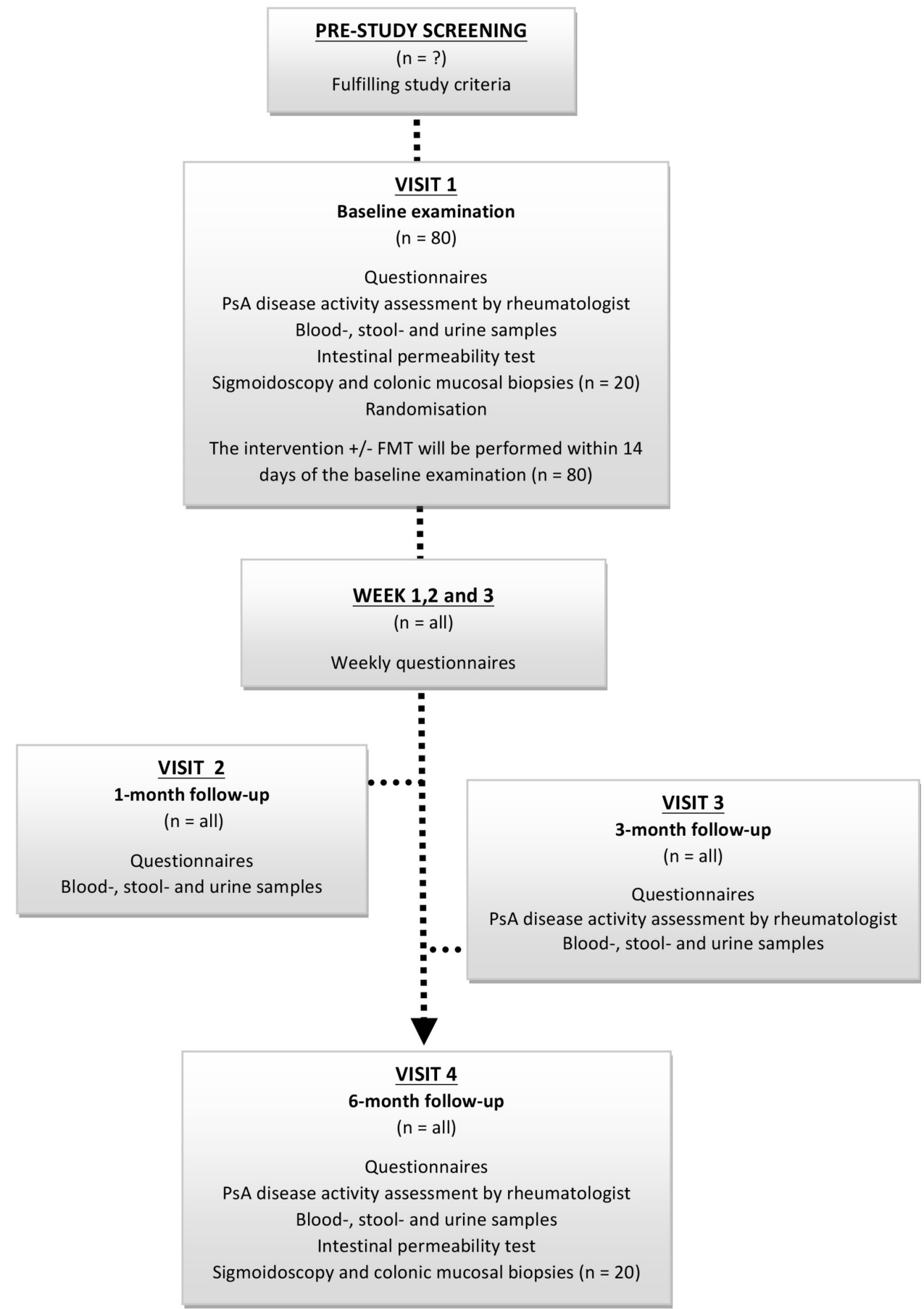

Figure 2 Participation timeline and characteristics of each visit. FMT, faecal microbiota transplantation; PsA, psoriatic arthritis.

\section{Inclusion criteria}

- Diagnosis of PsA according to the Classification Criteria for Psoriatic Arthritis. ${ }^{70}$

- Presence of active peripheral arthritis defined as $\geq 3$ swollen joints.

- Subcutaneously administered MTX treatment $(\geq 15 \mathrm{mg}$ /week (maximal tolerable dosage)) for a minimum of 3 months prior to study inclusion.

- Age 18-70 years.

Exclusion criteria

- Other inflammatory rheumatic diseases than PsA.
- Current axial disease activity or severe peripheral joint activity demanding immediate change of treatment or contraindicating placebo treatment for 6 months.

- IBD, coeliac disease, food allergy or other intestinal diseases.

- Current cancer or severe chronic infections.

- History of severe MTX toxicity or allergic reactions.

- Biological treatment within 6 months prior to inclusion.

- Non-MTX DMARD treatment within 3 months prior to inclusion. 
- Systemic and/or local intra-articular or peritendinous steroid injections within 3 months prior to inclusion.

- NSAIDs within 14 days prior to inclusion.

- Antibiotics within 3 months prior to inclusion.

- Pregnant or breastfeeding women.

- Not wishing to participate or unsuited for project evaluation.

\section{Stool donors}

The stool donor corps will consist of four anonymous (to the recipient) donors who must be healthy as assessed by a screening questionnaire, and be active members of the Danish blood donor corps, age $25-55$ years, body mass index between 18.5 and $25 \mathrm{~kg} / \mathrm{m}^{2}$ and an average alcohol intake $<7$ (women) or $<14$ (men) units per week. No alcohol intake within a week of donation is allowed, and no systemic medication including antibiotics and NSAIDs 6 months prior to the donation are allowed. The donor must eat a balanced diet (no extreme low-calorie or high-calorie diets), and must not be in a stressful life period. Before joining the stool donor corps, each potential donor will go through a screening process including stool analyses for faecal calprotectin and enteric pathogens (Aeromonas, Campylobacter, C. difficile, diarrhoeagenic Escherichia coli, Salmonella, Shigella, Vibrio, Yersinia enterocolitica and multidrug-resistant bacteria), parasites including microscopy of ova and cysts, Entamoeba histolytica/dispar (DNA), Cryptosporidium (DNA) and Giardia (DNA), sapovirus (RNA), rotavirus (RNA), human astrovirus (RNA), human adenoviruses (DNA) and noroviruses (RNA), a Helicobacter pylori breath test, blood tests for CRP) (acceptable level: $<6.0 \mathrm{mg} / \mathrm{L}$ ), white blood cell count (acceptable range: $3.50-8.80 \times 10^{9} / \mathrm{L}$ ), haemoglobin (acceptable range: $8.3-10.5 \mathrm{mmol} / \mathrm{L}$ ), albumin (acceptable range: 36-50 g/L), alanine aminotransferase (acceptable range: 10-70 U/L), estimated glomerular filtration rate (acceptable level: $>59 \mathrm{~mL} / \mathrm{min}$ ) and coeliac disease, and blood test for infectious agents including current infection with Epstein-Barr virus (IgM) and cytomegalovirus (IgM), hepatitis A, B, C and E, tuberculosis (QuantiFERON TB-Gold test), syphilis, HIV(ab HTLV1/2), E. histolytica (antibodies) and Strongyloides (antibodies), and a urine test for Chlamydia Trachomatis and Neisseria gonorrhoeae (DNA/RNA). After passing the screening tests, the donor will donate stool for the next month after which, the donor will have to pass the screening programme once more before the stool can be released for transplantation.

\section{Interventions}

\section{Overall study interventions}

The FMT will be an add-on strategy for patients with PsA with active joint disease despite ongoing treatment with weekly subcutaneously administered MTX. Therefore, all enrolled patients with PsA will continue their MTX treatment throughout the study, and they will remain on the same individual dosage that they received at the time of study inclusion (a minimum of $15 \mathrm{mg} /$ week cf. the patient inclusion criteria) in addition to folic acid supplement.
Paracetamol and tramadol in recommended dosages are allowed during the trial but no NSAIDs can be taken.

\section{Active and sham comparator}

Patients will be randomised into two groups with an allocation ratio of active-to-placebo treatment of 1:1. The active comparator group $(n=40)$ will have an FMT with healthy donor faeces-suspension $(250 \mathrm{~mL})$ containing $50 \mathrm{~g}$ donor faeces, saline $(\mathrm{NaCl} 0.9 \%)$ and glycerol $(10 \%)$, whereas the sham comparator group $(n=40)$ will be treated with an identical appearing sham procedure where the transplant solution will consist of $250 \mathrm{~mL}$ brown coloured (brown food colourant) isotonic saline $(\mathrm{NaCl} 0.9 \%)$.

\section{Preparing the FMT suspension}

Donors will collect faeces at home and transport it in a cooling bag to the study site within 1 hour. Faeces will be sieved to remove particulate material, followed by dilution in sterile saline $(0.9 \% \mathrm{NaCl})$ and $10 \%$ glycerol. The FMT suspension will be stored at $-80^{\circ} \mathrm{C}$ until use. On the day of the FMT transplantation, the transplant suspension $(250 \mathrm{~mL})$ will be thawed to $37^{\circ} \mathrm{C}$ and subsequently apportioned into five $50 \mathrm{~mL}$ syringes.

\section{FMT procedure}

The FMT will take place within 14 days (preferably 7 days) of the baseline clinical examination. The evening prior to the FMT, patients will take one dose $(40 \mathrm{mg})$ of oral proton-pump inhibitor. They will meet at the Department of Gastroenterology after a 6-hour fast. A total of $250 \mathrm{~mL}$ transplant suspension (active or placebo) will be installed in the duodenum using an oral-duodenal tube. The correct placement of the tube will be confirmed using gastroscopic guidance.

\section{Treatment strategy for non-responders}

Patients who present with increased or unacceptable disease activity during the 6-month trial period will, depending on the clinical presentation, be offered another treatment strategy, which may include local intra-articular steroid injections, change to another csDMARD or biological treatment. If the patient accepts such treatment changes, this will be characterised as FMT treatment failure according to the primary outcome definition (one intra-articular steroid injection is allowed).

\section{MTX toxicity and dropouts}

Blood tests for MTX toxicity will be performed in accordance with our current clinical practice. In case of MTX toxicity, severe side effects, pregnancy or occurrence of infectious disease or other diseases that contraindicate MTX treatment, MTX dosage will be decreased or the treatment will be paused. These patients will remain in the study (unless their condition contraindicates this), and they will be analysed as members of the treatment group to which they were randomised using intention-totreat-type analyses. 
Collection of faecal samples and metagenomics analysis

Fresh faecal samples will be collected by the patient at home using an EasySampler stool collection kit within 24 hours prior to the study visit. Samples will be stored in the patient's freezer until transported to the study site. During transport, samples will be kept on ice in a cooling bag. On arrival to the study site, samples will immediately be transferred to the biobank and stored at $-80^{\circ} \mathrm{C}$. Bacterial DNA will be extracted from the faecal samples following established standard protocols including bead beating using a NucleoSpin soil kit (Macherey-Nagel, Germany) according to manufacturer's instructions. DNA will be sequenced using the BGISEQ-500 Platform, which was recently benchmarked against the Illumina platforms showing excellent intraplatform reproducibility and less GC bias than observed using the Illumina platforms. ${ }^{71}$ The faecal metagenomics bioinformatics analyses will be performed using comprehensive pipelines including the assembly of metagenomics linkage groups/metagenomics species, ${ }^{72} 73$ taxonomic annotation and extensive functional analyses based on metagenomic species, which provides a superior dataset compared with the conventional analyses based on the total gene pool. ${ }^{74}$

\section{Intestinal permeability test}

After an overnight fasting, patients will provide a urine sample before ingesting $100 \mathrm{~mL}$ water containing $10 \mathrm{~g}$ of lactulose and $5 \mathrm{~g}$ of D-mannitol. All the urine passed in the subsequent 5 hours will be collected into a $2 \mathrm{~L}$ plastic container containing $1 \mathrm{~mL}$ of chlorohexidine (20 mg/ $\mathrm{mL}$ ) as a preservative. After 3 and 5 hours, the volume of the urine will be measured and a small volume $(10 \mathrm{~mL})$ will be preserved and stored at $-80^{\circ} \mathrm{C}$ until analysis. No food or drinking (except for water) will be allowed during the test. $^{75} 76$

\section{Outcomes}

Primary outcome measure

Treatment failure (time frame: 6 months $( \pm 14$ days $)$ ).

Proportion of patients in each group who experience treatment failure according to shared decision-making between patient and rheumatologist defined as at least one of the following:

- Need for more than one intra-articular glucocorticoid injection due to disease activity.

- Need for change to other csDMARDs (eg, oral leflunomide or sulfasalazine) according to the updated Danish treatment guideline due to disease activity.

- Need for biologic treatment according to the updated Danish treatment guideline due to severe disease activity.

\section{Secondary outcome measures}

Change from baseline in the Short (2-page) $\mathrm{HAQ}^{77} 78$ (time frame: baseline, 1 week, 2 weeks, 3 weeks, 4 weeks, 3 months ( \pm 7 days $), 6$ months $( \pm 14$ days $))$.
Change from baseline in the Dermatology Life Quality Index Questionnaire $^{79}$ (time frame: 1 week, 2 weeks, 3 weeks, 4 weeks, 3 months ( \pm 7 days), 6 months ( \pm 14 days)).

Changes from baseline in patient reported gastrointestinal side effects (time frame: 1 week, 2 weeks, 3 weeks, 4 weeks, 3 months ( \pm 7 days $), 6$ months $( \pm 14$ days $))$.

Other non-gastrointestinal patient reported side effects (time frame: 1 week, 2 weeks, 3 weeks, 4 weeks, 3 months ( \pm 7 days), 6 months ( \pm 14 days $)]$.

Proportion of patients in each group achieving the American College of Rheumatology (ACR) ${ }^{80}$ Response Criteria (time frame: 3 months ( \pm 7 days), 6 months $( \pm 14$ days)):

I. ACR20 response criteria ${ }^{81}$

II. ACR50 response criteria ${ }^{82}$

III. ACR70 response criteria. ${ }^{82}$

Proportion of patients in each group achieving the Psoriatic Arthritis Response Criteria ${ }^{80}$ (time frame: 3 months ( \pm 7 days), 6 months ( \pm 14 days $))$.

Change from baseline in the Spondyloarthritis Research Consortium of Canada Enthesitis Index ${ }^{68}$ in the subset of patients who have enthesitis at baseline (time frame: 3 months ( \pm 7 days $), 6$ months ( \pm 14 days $)$ ).

Change from baseline in the Psoriasis Area Severity Index (PASI $)^{83}$ in the subset of patients who have skin psoriasis at baseline (time frame: 3 months ( \pm 7 days), 6 months $( \pm 14$ days $))$.

Change from baseline in the number of digits affected with dactylitis in the subset of patients who have dactylitis at baseline (time frame: 3 months ( \pm 7 days), 6 months $( \pm 14$ days $))$.

Number of adverse events (AEs) in each group (time frame: 6 months $( \pm 14$ days $))$.

Number of AEs in each group leading to discontinuation (time frame: 6 months $( \pm 14$ days $)$ ).

Number of patients with at least one AE in each group (time frame: 6 months $( \pm 14$ days $)$ ).

Tertiary (exploratory secondary) outcomes: proportion of patients in each group achieving changes in plasma CRP, changes in tender point count, ${ }^{84}$ changes in faecal bacteria composition and metabolism, changes in intestinal permeability, changes in plasma orosomucoid, changes in plasma and faecal calprotectin, ${ }^{85}$ changes in serum 1,25-dihydroxyvitamin $\mathrm{D}$, changes in cardiovascular risk factors including body mass index, blood pressure, plasma triglyceride, plasma low-density lipoprotein-cholesterol, plasma high-density lipoprotein-cholesterol, plasma total-cholesterol and haemoglobin A1c levels, changes in specific circulating inflammatory markers (ie, cytokines, adipokines and chemokines) and macroscopic and microscopic inflammatory changes of the colonic mucosa (see table 1).

\section{Safety}

The most frequently reported adverse events (AEs) related to FMT are vomiting, belching, mild diarrhoea, abdominal cramping, transient fever and elevated CRP on the day of the procedure. ${ }^{86}$ A recent systematic review 
Table 1 Protocol schedule of forms and procedures.

\begin{tabular}{|c|c|c|c|c|c|c|}
\hline Activity/assessment & $\begin{array}{l}\text { Prestudy } \\
\text { screening }\end{array}$ & $\begin{array}{c}\text { Visit 1 } \\
\text { Baseline }\end{array}$ & $\begin{array}{c}\text { Weeks } \\
1,2 \text { and } 3\end{array}$ & $\begin{array}{c}\text { Visit } 2 \\
1 \text { month }\end{array}$ & $\begin{array}{c}\text { Visit } 3 \\
\text { 3months }\end{array}$ & $\begin{array}{c}\text { Visit } 4 \\
6 \text { months }\end{array}$ \\
\hline Patients & $\mathrm{n}=?$ & $\mathrm{n}=80$ & $\mathrm{n}=$ all & $\mathrm{n}=$ all & $\mathrm{n}=$ all & $\mathrm{n}=$ all \\
\hline Inclusion/exclusion form & $x$ & & & & & \\
\hline Consent form & & $x$ & & & & \\
\hline Patient global (VAS 0- 100 mm) & & $x$ & $x$ & $x$ & $x$ & $x$ \\
\hline Patient fatigue (VAS 0-100 mm) & & $x$ & $x$ & $x$ & $x$ & $x$ \\
\hline Patient pain (VAS 0-100 mm) & & $x$ & $x$ & $x$ & $x$ & $x$ \\
\hline $\mathrm{HAQ}$ & & $x$ & $x$ & $\mathrm{x}$ & $x$ & $x$ \\
\hline Gastrointestinal symptom diary & & $x$ & $x$ & $\mathrm{x}$ & $x$ & $x$ \\
\hline Eating habits questionnaire & & $x$ & & & & \\
\hline
\end{tabular}

Clinical examination

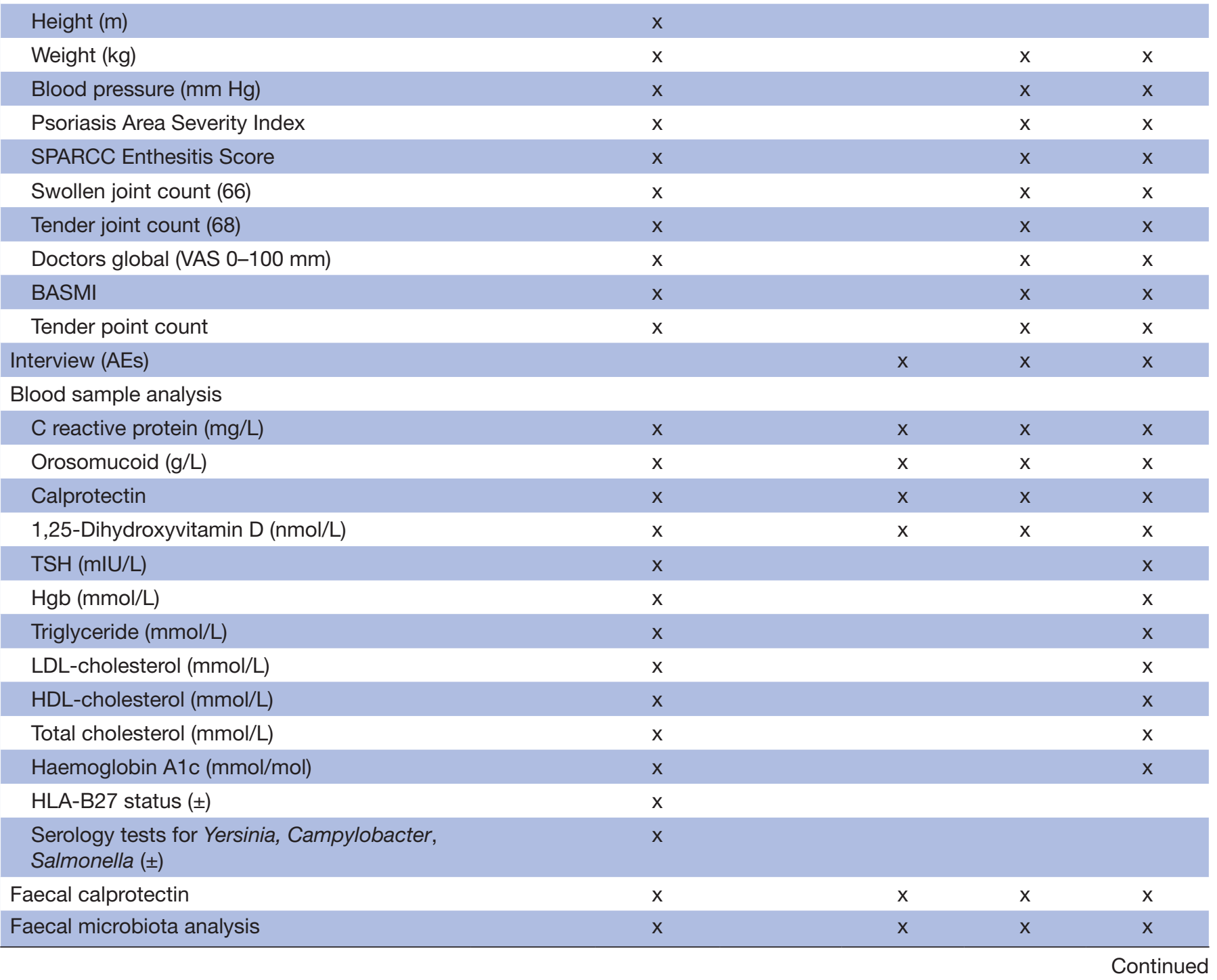


Table 1 Continued

\begin{tabular}{|c|c|c|c|c|c|c|}
\hline Activity/assessment & $\begin{array}{l}\text { Prestudy } \\
\text { screening }\end{array}$ & $\begin{array}{c}\text { Visit } 1 \\
\text { Baseline }\end{array}$ & $\begin{array}{c}\text { Weeks } \\
1,2 \text { and } 3\end{array}$ & $\begin{array}{c}\text { Visit } 2 \\
1 \text { month }\end{array}$ & $\begin{array}{c}\text { Visit } 3 \\
3 \text { months }\end{array}$ & $\begin{array}{c}\text { Visit } 4 \\
6 \text { months }\end{array}$ \\
\hline Sigmoidoscopy and mucosa biopsy & & $x$ & & & & $x$ \\
\hline Intestinal permeability test & & $\mathrm{x}$ & & & & $\mathrm{x}$ \\
\hline Intervention ( $\pm \mathrm{FMT})$ & & $x$ & & & & \\
\hline
\end{tabular}

AE, adverse event; BASDAI, Bath Ankylosing Spondylitis Disease Activity Index; BASFI; Bath Ankylosing Spondylitis Functional Index; BASMI, Bath Ankylosing Spondylitis Metrology index; DLQI, Dermatology Quality of Life Index; HAQ, Health Assessment Questionnaire; HDL, highdensity lipoprotein; Hgb, haemoglobin; LDL, low-density lipoprotein; SPARCC, Spondyloarthritis Research Consortium of Canada; TSH, thyroid-stimulating hormone; VAS, visual analogue scale.

on the AEs of FMT identified 50 relevant studies with a total of 1089 patients. In this review, the incidences of serious adverse events (SAEs) for FMT were $2.0 \%$ and $6.1 \%$ for upper and lower gastrointestinal routes, respectively. The SAEs that probably or possibly were related to FMT included infections $(0.7 \%)$, IBD flare $(0.6 \%)$, death $(0.3 \%)$, autoimmune diseases and FMT procedure-related injury. ${ }^{87}$ Although most of the patients included in this review suffered from severe gastrointestinal diseases (C. difficile infection and/or IBD), these findings warrant caution when performing FMT, especially when introducing the procedure in a new patient population. In addition, the potential long-term side effects following FMT remains largely unknown. ${ }^{8}$ Still, when strict donor screening is conducted and the procedure is performed by experienced practitioners, FMT is in general considered safe, and even elderly patients with a poor medical condition and multiple comorbidities as well as immunosuppressed patients have been proven to tolerate the FMT procedure well. ${ }^{89-93}$

In the present study, we will carefully monitor and evaluate safety by means of open assessment of AEs. All reported or observed AEs are recorded by the investigators, and will be monitored until resolution, stabilisation or until it has been shown that the study intervention is not the cause. The National Cancer Institute Common Terminology Criteria for Adverse Events, V.4.03 (NIH publication \# 09-7473), will be used to grade the severity of AEs. Gastrointestinal side effects (nausea, vomiting, abdominal pain, number of stools per week, stool type (Bristol Stool Chart), blood or mucus in the stool) will be registered by the patients once a week for the first month following the randomised intervention. Routine blood screening for MTX toxicity will normally be performed at weeks $4,10,16,22$ but can be more frequent if decided by the responsible treating rheumatologist depending on symptoms or signs of MTX toxicity. Subject incidence rates of all treatment-emergent AEs will be tabulated by system organ class and preferred term. Tables of fatal AEs, SAEs, AEs leading to withdrawal from study and significant treatment-emergent AEs, will also be provided. For the long-term extension portion of this study, exposure-adjusted event rates will be summarised.

\section{Sample size and power considerations}

When designing this trial, no prior data for FMT efficacy in patients with rheumatic disease were available. However, we found it reasonable to assume that if patients with rheumatic disease should be willing to receive FMT as a future standardised treatment, the procedure should at least provide an effect size well beyond a moderate effect size. Consequently, we decided that at least twice as many patients with PsA in the sham group should be treatment failures compared with the FMT group if the procedure should be considered clinically relevant. For a comparison of two independent binomial proportions using the Pearson's $\mathrm{X}^{2}$ statistic with a $\mathrm{X}^{2}$ approximation (a two-sided significance level of 0.05), a sample size of 40 patients with PsA per group has a power of 90\% (0.895), if we assume that the proportions of treatment failures are $35 \%$ (FMT-active group) and 70\% (FMT-sham group), respectively. Consequently, the inclusion of 80 patients with PsA allocated (1:1) to two treatment arms is believed to be sufficient to reveal any difference of clinical importance between treatment groups (ie, an Number needed to treat (NNT) $<3$ patients).

Assuming that there will be some attrition during the 6-month trial period, we also estimated how much dropout would be possible while still having a reasonable statistical power $(80 \%)$ : a total sample size of 62 patients with PsA assuming a comparable level of withdrawals (31 patients completing in each group) achieves a power of at least 0.8 with the proportion of treatment failures indicated above, that is, even if we experience a dropout rate of $20 \%$, our trial will have $80 \%$ chance of detecting the intentional difference between groups.

Beyond the primary end point, a total sample size of 80 (with a balanced design) corresponds to a sufficient statistical power $(82 \%)$ to detect a standardised mean difference of 0.65 SD units (ie, Cohen's effect size) in any of the patient-reported outcome measures.

\section{Randomisation, allocation concealment and blinding}

The randomisation has been conducted using central-computer randomisation. Patients are randomly allocated (1:1) to receive either an FMT or a placebo saline transplant (sham procedure). The randomisation lists were 
generated by the trial statistician and uploaded to the REDCap database by an independent data manager who is not involved in any other aspects of the trial. Eligible patients will, after signing informed consent, be assigned randomly in permuted blocks with varying sizes of 4 and 6 , according to computer-generated random numbers (SAS programming via SAS PROC PLAN), to undergo either FMT or saline (sham) procedure using stratification for centre. The randomisation of each patient will be implemented by the local trial coordinator and allocation will be concealed as this is done independent of the predetermined sequence generation (ie, randomisation). The patients, care providers and outcome assessors will remain unaware of the group assignments, and only de-identified codes will be used to link participants to their data during the study to maintain their confidentiality. In case of exceptional circumstances when knowledge of the treatment allocation is essential for further management of the patient, the trial secretary will reveal the assigned intervention to the treating doctor. However, patients, trial care providers and outcome assessors will remain blinded as far as possible. Cases of unblinding will be registered and reported.

\section{Data collection, management and confidentiality}

Data will be entered via a secure web-based electronic clinical report form into a central REDCap ${ }^{94}$ database hosted by Odense Patient data Explorative Network at Odense University Hospital. Data obtained during the clinical examination will be entered directly into the database. Also, patient questionnaires will be fulfilled directly into the database. Access to the study data will be restricted, and a password system will be used to control access. All information about the patients' health and other private matters is covered by confidentiality. The authorisation from the Danish Data Protection Agency has been secured.

\section{Statistical methods}

The full analysis set will consist of all randomised participants (ie, the intention-to-treat (ITT) population): participants will be analysed according to their randomised treatment group, that is, the ITT has the consequence that participants allocated to a treatment group will be followed up, assessed and analysed as members of that group irrespective of their compliance to the planned treatment. The safety analysis set will include all patients who were randomly assigned to a study group and had exposure to a transplant (independent of group). Descriptive statistics will be provided for demographics and baseline characteristics. The summary statistics of continuous variables will include: $\mathrm{N}$, mean, $\mathrm{SD}$, median, interquartiles and range. All summaries presenting frequencies and incidences will include counts, percentages and the total number of participants in the corresponding arm.

The prespecified efficacy analyses will be based on data from the full-analysis set, which include all patients who underwent randomisation, have had their baseline measurement performed and who have received the initial transplant (independent of group). Although proper random assignment prevents selection bias, it does not guarantee that the groups will be equivalent at baseline. Any differences in baseline characteristics are, however, the result of chance rather than bias ${ }^{95}$; thus, the study groups will be evaluated (and presented) at baseline for important demographic and clinical characteristics so that readers can assess how similar they are. However, only cohort studies can be subject to selection bias and confounding due to differences in baseline characteristics between the intervention and comparison groups. ${ }^{96}$

Our strategy for ITT analysis with incomplete observations will be based on the recommendations from White et $a \ell^{97}$ :

1. Attempt to follow-up all randomised participants, even if they withdraw from allocated treatment.

2. Perform a main analysis of all observed data (data as observed).

3. Perform sensitivity analyses to explore the effect of departures from the assumption made in the main analysis (Baseline Observation Carried Forward (BOCF) imputations, repeated measures mixed models and multiple imputations).

This results in the following steps: missing values will be imputed with the use of a non-responder imputation by use of the BOCF method for measurements made after baseline. Thus, missing data for dichotomous endpoints will also be imputed using a conservative 'null responder' imputation, assuming the patient did not have any benefit from being enrolled in the trial (eg, for the primary end point we will assume that the patient had a treatment failure which is valid based on clinical judgement even if data are not missing at random). Other sensitivity analyses will be including 'worst' and 'best' case imputation, repeated-measures and multiple-imputation analyses, using model-based approaches; repeated measures linear mixed models will also be used to model the potential group-dependent trajectories over time (ie, repeated mixed models and multiple imputation are valid if data are assumed missing at random).

Categorical data for dichotomous end points will be analysed with the use of logistic regression with the model including treatment and centre as class effects. For continuous outcome measures, analysis of covariance models will be used to analyse mean changes in continuous end points. All models will include treatment, centre, with the baseline value of the relevant variable as covariates.

Additionally, completer analyses will be performed on those who complete 6 months of treatment. During follow-up, any medical treatments which could potentially modify the intestinal microbiota including antibiotics will be reported, but will not affect the statistical analysis. Statistical estimates will be calculated as ORs for the dichotomous variables and difference between means for continuous outcomes reported with 95\% CIs. Two-sided 95\% CIs and $\mathrm{P}$ values for primary, secondary and exploratory outcomes will be computed and will not be adjusted 
for multiplicity, but will be interpreted cautiously as this is an exploratory trial per se.

Prespecified exploratory analyses: stratified analyses will investigate whether the treatment effect varies with i) the faecal microbiota analyses performed at follow-up compared with baseline ( \pm long-term changes in the intestinal microbiota and intestinal inflammation) and ii) the demographic match (sex, age) between the stool donor and the recipient. Non-responders will represent the outcome group not fulfilling the primary outcome measure. Differences in demographics and baseline disease activity between this treatment-failure subpopulation and the remaining group will be examined to identify potential prognostic factors for poor responders. Patients not participating in the follow-up examination will be classified as 'dropouts', and if possible, the reason for not participating will be registered.

Metagenomics analysis will be performed as described in the section on collection of faecal samples. ${ }^{72} 73$ 74 identify possible associations, metagenome analysis will be correlated to all clinical parameter. We will use an L1 restricted least absolute shrinkage and selection operator (LASSO) procedure to determine the optimal number of features to be tested as described. Analysis of correlations between microbiota taxonomic or functional features, community diversity indices and sample metadata variables will be performed using Spearman's correlation tests corrected for multiple tests using the Benjamini-Hochberg false discovery rate control procedure. To control for confounders, we will use blocked Spearman's correlation tests. ${ }^{989}$

Data will be analysed with the STATA statistical package (V.15; StataCorp), and SAS software (V.9.4; SAS Institute, Cary, North Carolina, USA).

\section{ETHICS AND DISSEMINATION}

This study is designed as a proof-of-concept clinical trial and will be performed in agreement with Good Clinical Practice (GCP) standards, and in accordance with the ethical standards of the responsible committee on human experimentation (institutional and national) and with the Helsinki Declaration (64th, 2013). The relevance of the study, the design and the recruitment strategy were evaluated with three patient research partners (PRPs), and alterations especially in primary outcome and recruitment strategy were embedded. Furthermore, a minimum of two PRPs (participating in the study) will be involved in the discussion regarding the progress of the recruitment phase and results, and will be offered the opportunity to comment on the manuscript draft. The trial has been registered with ClinicalTrials.gov (NCT03058900) and important protocol modifications will be updated here. The Danish Health and Medicines Authority does not classify the FMT procedure as a medical intervention, and has had no objection to the use of FMT for this study and patient category. Thus, no GCP auditing is legally required. A report describing any potential side effects and AEs will be submitted to the Ethics Committee yearly. Suspected Unexpected Serious Adverse Reactions will be reported to the Ethics Committee within 7 days. Based on these reports, the Ethics committee can determine to terminate the trial early. The Danish Patient Compensation Association provides compensations for patients injured in connection to medical clinical trials.

Although the Danish Health Authorities, for the time being, do not classify donor faecal microbiota as tissue, all steps of the stool donor recruitment, stool donation and FMT preparation will be in accordance with the Danish Tissue Law to ensure that the quality and safety standards laid down in the Danish Legislation BEK nr 764 of 26 May 2015 (implementing Directive 2004/23/EC) are met. Four stool donors will be recruited from the South Danish Transfusion Service \& Tissue Centre, Department of Clinical Immunology, Odense University Hospital, and they will be carefully screened for potentially transmissible infections and other conditions associated with gut microbiota function before their stool can be released for FMT. Being a stool donor is voluntary, and no compensation fee will be given. Furthermore, to ensure donor traceability, each patient in the active treatment arm will only receive microbiota from one donor. Also, frozen samples will be clearly labelled with a unique donation code based on the ISBT 128 coding and labelling system, and the release of the final product will adhere to the standards for tissue and blood donation.

Due to the well-documented risk of permanent joint destruction and occurrence of extra-articular manifestations in the PsA disease course, identification of new treatment modalities and biomarkers is essential to help the physician to slow down the disease development or ultimately to prevent it. All patients with PsA participating in this study have significant activity in their joint disease despite treatment with the current guideline treatment and first-line drug, MTX, for this condition. This patient population will therefore benefit greatly from new treatment options. Consequently, when weighing the pros and cons, this trial should be performed from a scientific and ethical perspective.

Dissemination will occur through presentations at national and international conferences and publications in international peer-reviewed journal(s).

\section{DISCUSSION}

Recent years have seen growing recognition of the complexity of the role of the microbiota in shaping the immune system and its potential effects for health and disease. ${ }^{22} 100101$ In particular, the gut bacterial composition has been associated with the pathogenesis of autoimmune and inflammatory diseases. ${ }^{102-105}$ Intriguingly, an abnormal intestinal bacterial composition has been observed in patients with PsA, and this association has fostered theories linking intestinal dysbiosis and PsA joint inflammation. ${ }^{106}$ Still, it remains to be elucidated whether the intestinal dysbiosis and rheumatic diseases are causally 
related, ${ }^{55}$ and if so, whether dysbiosis is an inciting event in the inflammatory process or a consequence of local and/or systemic inflammation. ${ }^{54}{ }^{107}$ We expect that this double-blind, randomised, placebo-controlled trial will shed new light on this highly relevant topic.

This is the first time that the efficacy and safety of FMT is being investigated in MTX immunosuppressed patients with rheumatic diseases. Subsequently, no data on the feasibility of conducting FMTs in the rheumatological setting is, so far, available. Nor do we know whether one FMT will be sufficient, or whether it should be repeated shortly after the first intervention to normalise the alterations of the intestinal microbiota, which would expectedly enhance any potential anti-inflammatory effects. In the present proof-of-concept clinical trial, the FMT procedure is considered an add-on to the current guideline intervention and first-line drug, MTX. Therefore, from a pragmatic and ethical perspective, we have decided to perform only one FMT (or sham procedure) in each patient even if we are well aware that this approach may not be adequate to achieve long-lasting effects. Indeed, in patients with IBDs it appears that performing a frequent dosing regime repeating the FMT procedure up to five times a week for 8 weeks provided the best results. ${ }^{51} 108109$ Hence, in contrast to the treatment of C. difficile infections where the microbiota is pushed past the point of homeostasis and can be restored following only one $\mathrm{FMT}^{47}$ the chronic nature of PsA and other autoimmune and inflammatory diseases, and the somewhat lesser degree of intestinal dysbiosis, may make the host microbiota more resistant to long-lasting modifications. Nevertheless, we hope that the FMT procedure in the present study will be sufficient to boost the effects of MTX so that the participants who are all MTX non-responders prior to study enrolment will achieve disease control without needing to add or switch to other non-MTX medication.

In the present trial, the primary outcome measure is defined as the occurrence of treatment failure according to shared decision-making between patient and physician evaluated at 6 months following the randomised intervention (FMT vs sham procedure). Shared decision-making is a process in which both patient and health professional make a decision, taking into account the best evidence of available treatment options and the patient's values and preferences. This approach is considered a key element in the management of rheumatic diseases. ${ }^{110}$ As both patients and the treating rheumatologists are blinded to the randomised intervention, the shared decision-making will be unaffected by the type of transplant suspension (active or placebo) installed at baseline. Nevertheless, we acknowledge that our assumption that twice as many patients with PsA in the sham group will be treatment failures is ambitious, and that we might miss a smaller and less clinically significant treatment effect of the FMT procedure. In this case, we hope that our secondary outcome measures will be able to detect potential trends of positive effects in PsA subdomains such as enthesitis score, dactylitis count and PASI skin score. In addition to the primary end point evaluation at 6 months, patients will be asked to fill out a weekly questionnaire regarding side effects as well as skin and arthritis symptoms during the first month following the randomised intervention to reveal any short-term effects on patient-reported outcomes.

Next, only patients with active peripheral PsA will be included. One reason for this is that this will be the first time that FMT is performed on patients with rheumatic disease. Therefore, it seems reasonable only to enrol patients who have had inadequate effect from the initial guideline treatment (MTX), and consequently, on an individual basis could benefit the most from participating in new experimental clinical trials. Also, since patients need to have at least three swollen joints, we expect that we will be able to detect treatment effects of clinical importance. The fact that we do not include recent-onset treatment-naïve patients will, of course, limit our ability to generalise our findings unto the entire patient population with PsA. Indeed, in a recent randomised controlled trial of FMT in patients with ulcerative colitis, participants with a recent diagnosis $(<1$ year) were statistically significantly more likely to respond to FMT compared with those with longer disease duration. ${ }^{108}$ That patients will have to subcutaneously administer MTX for at least 3 months prior to study enrolment will ensure that low intestinal MTX absorption is excluded as a potential effect modifier for the poor MTX response. In addition, as many drugs, including MTX, seem to affect the intestinal microbiological milieu, ${ }^{111-114}$ bypassing the intestine during MTX administration will ensure that no local non-disease-related effects on the intestinal microbiota will occur.

A great challenge when conducting a trial of FMT is that for the present being there is a lack of both national and international recommendations guiding the regulation and the best clinical practices for donor screening, stool sample handling and preparation of the FMT suspension. ${ }^{115-117}$ Indeed, the variability in faecal bacterial communities can complicate or undermine treatment efficacy. This variability stems from both biological variation and variation introduced by sample handling. A recent study reported that oxygen exposure degraded faecal bacterial communities, whereas freeze-thaw cycles and lag time between donor defaecation and transplant preparation had much more limited effects. ${ }^{118}$ Given that many intestinal bacteria are obligate anaerobe, including many beneficial bacteria potentially possessing anti-inflammatory effects, exposure to oxygen during the preparation of FMT may potentially compromise the therapeutic value of FMT in PsA and other inflammatory diseases. Therefore, although frozen faecal preparations of stool suspended into physiological saline and glycerol have proven just as effective as fresh stool in treating $C$. difficile infections, ${ }^{119}$ the optimal transplant preparation method in treating inflammatory diseases remains to be established.

Our stool handling setup is in line with the prevailing practice, which includes mixing and filtration of the stool 
suspension and adding saline and glycerol as a cryopreservative before storage at $-80^{\circ} \mathrm{C} .{ }^{117}$ In addition, we have sought to limit the oxygen exposure during transport by placing the donor stool within a plastic bag, which is subsequently put into a tightly closed small plastic container. Supplementary, during preparation the solution will not be homogenised for more than $10 \mathrm{~s}$. Nevertheless, the lack of optimal anaerobe conditions during stool handling could possibly undermine the therapeutic potential of our FMT procedure. Furthermore, although we aim to use $50 \mathrm{~g}$ of faeces for each transplant, we acknowledge that the exact weight between donations could vary with an estimated $\pm 5 \mathrm{~g}$. Also, due to the wide variability in microbial content in stool between donations, the content cannot be fully standardised, and may likely differ between each FMT procedure. However, to meet this challenge we will collect and store samples from each donation which will enable us to determine the microbiota composition of each donation in case some donations prove more effective than others.

Stool donor selection is another critical issue that needs to be addressed. The composition of the normal microbiota composition has only recently been mapped, ${ }^{74}$ and the existence of a limited number of well-balanced host-microbial symbiotic states, where one or more bacteria species are considered the main functional driver(s), have been identified using clustering of metagenomic sequences. ${ }^{120}$ Still, the most favourable donor microbiota composition for treating inflammatory diseases has yet to be determined. Therefore, it also remains to be established whether donors with a high stool bacteria diversity should be preferred over isolation of specific bacteria, or if pooled stool samples from several donors outperforms a single-donor transplant. ${ }^{5121}$ We have chosen to use only single donations from four different anonymous stool donors to ensure donor traceability and to enable us to identify any individual donor-specific microbial effects. Also, since host intrinsic, environmental and dietary factors as well as pharmaceutical drugs have been associated with gut bacterial composition and functionality, ${ }^{111} 112122123$ the donors must eat a balanced diet, not be overweight or take any medications or be physically or psychologically stressed, smoke or consume alcohol during the donation period to limit the risk of transferring 'abnormal' microbiota to the recipients. These donor criteria have been set for safety reasons, and we acknowledge that this could potentially limit the interdonor microbiota diversity due to shared lifestyle characteristics.

Another factor to keep in mind is the concept of matching donor and recipient, which may be of importance for enhancing the colonisation capabilities of the donor microbial communities. In fact, Rossen $e t a l^{109} \mathrm{did}$ find that in patients with ulcerative colitis, the microbiota of FMT responders shifted to their respective donors, whereas non-responders did not. Li et al ${ }^{124}$ reported that donor bacteria strains established extensively in the recipient and persisted for at least 3 months with a negligible decline of donor-strain populations detected between 45 days and 3 months following FMT in patients with metabolic syndrome.
However, they also found that recipients receiving the same donor transplant displayed varying degrees of microbiota transfer, indicating individual patterns of microbiome resistance and donor-recipient compatibilities. In addition, host genetics is known to effect the gut microbiota, ${ }^{125}$ and animal models have shown that sex ${ }^{126}$ and age ${ }^{127}$ also can be potentially modifiers of the gut bacteria composition. These observations may prove to be of importance for the outcome of FMT in inflammatory diseases. ${ }^{128}$ However, whether sex-matching and/or age-matching between donor and recipient is crucial for a successful FMT in humans remains to be enlighten. Therefore, in the present study, no donor-recipient matching will be conducted. However, a subgroup analysis will be performed to reveal any trend that could indicate better results in sex-matched or age-matched cases.

Furthermore, as the interactions between the microbiota and the host are influenced by cooperation and competition between pathogenic and commensal microbes and multiple environmental variables, the lifestyle of the recipient following the FMT may be of importance. Nevertheless, due to the uncertainty of how to define the optimal lifestyle and the lack of knowledge on how different lifestyle factors may interfere with the microbiota, we have decided that the patients in the present study will not have to adhere to any predefined lifestyle 'regime' or diet following the randomised intervention. However, every participant will fulfil an eating habit questionnaire at the beginning of the trial.

Finally, non-bacteria microorganisms such as bacteriophages, viruses and fungi may also be of importance when targeting components of the microbiota or host cells for therapeutic purposes. ${ }^{129-131}$ Other complicating factors may include the composition of other microbiological niches such as the oral, lung, genitourinary and skin microbiota. ${ }^{132} 133$ Indeed, the latter could likely prove to be of significance in patients with skin psoriasis. However, these factors will not be assessed in the present study.

In conclusion, this trial has the potential to substantially expand the growing body of literature on the role of the intestinal microbiota in general and in PsA in particular. Furthermore, we anticipate that this study will enhance our understanding of cause and effect. The results of this study, when completed, may be exploited for biomarker discovery, and for diagnostic and therapeutic purposes.

\section{Author affiliations}

${ }^{1}$ Department of Rheumatology, Odense University Hospital, Odense, Denmark ${ }^{2}$ Odense Patient data Explorative Network (OPEN), Department of Clinical Institute, University of Southern Denmark, Odense, Denmark

${ }^{3}$ Department of Gastroenterology, Odense University Hospital, Odense, Denmark ${ }^{4}$ Department of Clinical Microbiology, Odense University Hospital, Odense, Denmark ${ }^{5}$ Department of Clinical Immunology, Odense University Hospital, Odense, Denmark ${ }^{6}$ Diagnostic Centre, Silkeborg Regional Hospital, Silkeborg, Denmark ${ }^{7}$ IRS-Centre Sonderjylland, Hospital of Southern Jutland, Aabenraa, Denmark ${ }^{8}$ Institute of Molecular Medicine, University of Southern Denmark, Odense, Denmark ${ }^{9}$ Laboratory of Genomics and Molecular Biomedicine, Department of Biology, University of Copenhagen, Copenhagen, Denmark 
${ }^{10}$ Institute of Metagenomics, BGI-Shenzhen, Shenzhen, China

${ }^{11}$ Musculoskeletal Statistics Unit, Parker Institute, Frederiksberg and Bispebjerg Hospital, Copenhagen, Denmark

Acknowledgements The authors would like to thank Lene Albjerg, biomedical laboratory technologist and quality control manager at the Department of Clinical Immunology, Odense University Hospital, Denmark, for her expertise and dedicated work regarding the practical handling of the FMT suspension.

Contributors TE, MSK, RC and JK conceived and developed the idea for the study. TE and MSK are the principal investigators and wrote the first study protocol draft. TE and MSK were responsible for all communication with the scientific ethical committee, the Danish Data Protection Agency and the National Health Board. TE is the responsible party and sponsor. MSK, TE, HCH, JKP, HLM and UF sat up the inclusion and exclusion criteria for the patients with psoriatic arthritis, and the latter five rheumatologists are conducting the clinical examinations. JK, FMP and HG discussed and planned the FMT procedure, and they are conducting the FMTs and the sigmoidoscopies (obtaining mucosae tissue samples). DKH and HMH helped set up the donor screening programme, and they were responsible for conducting this programme and performing the microbiological and immunological tests. VA and KK are responsible for the omics and microbiome analyses, and have advised on how the tissue collection should be performed and what kind of tissue would be relevant to collect. RC has written the statistical analysis plan and will be responsible for the final statistical analyses. In conclusion, all participants designated as authors have contributed to the conception and design of the study, and they have critically either drafted or revised the first draft of the study protocol and the protocol paper. Also, all authors have approved the final version before submission.

Funding This work was supported by the Region of SouthernDenmark, Odense University Hospital, the Danish Regions, University of SouthernDenmark, the Danish Rheumatism Association, the Danish Psoriasis Research Fund, Odense Patient Explorativedata Network (OPEN), and Fabrikant Vilhelm Pedersen and Hustrus Legat based on the recommendation of the Novo Nordisk Foundation. Musculoskeletal Statistics Unit at the Parker Institute, Bispebjerg and Frederiksberg Hospital(R. Christensen), is supported by a core grant from the 0ak Foundation (OCAY-13-309). K. Kristiansen is supported by BGI-Research, BGI-Shenzhen, China.

Competing interests None declared.

Patient consent Not required.

Ethics approval The study protocol has been approved by the Regional Committees on Health Research Ethics for Southern Denmark (DK-S-20150080) and the Danish Data Protection Agency (15/41684).

Provenance and peer review Not commissioned; externally peer reviewed.

Open Access This is an Open Access article distributed in accordance with the Creative Commons Attribution Non Commercial (CC BY-NC 4.0) license, which permits others to distribute, remix, adapt, build upon this work non-commercially, and license their derivative works on different terms, provided the original work is properly cited and the use is non-commercial. See: http://creativecommons.org/ licenses/by-nc/4.0/

(C) Article author(s) (or their employer(s) unless otherwise stated in the text of the article) 2018. All rights reserved. No commercial use is permitted unless otherwise expressly granted.

\section{REFERENCES}

1. Scher JU, Littman DR, Abramson SB. Review: microbiome in inflammatory arthritis and human rheumatic diseases. Arthritis Rheumatol 2016;68:35-45.

2. Asquith $\mathrm{M}$, Elewaut $\mathrm{D}$, Lin $\mathrm{P}$, et al. The role of the gut and microbes in the pathogenesis of spondyloarthritis. Best Pract Res Clin Rheumatol 2014;28:687-702.

3. Stoll ML. Gut microbes, immunity, and spondyloarthritis. Clinical Immunology 2015;159:134-42.

4. Costello ME, Ciccia F, Willner D, et al. Brief report: intestinal dysbiosis in ankylosing spondylitis. Arthritis Rheumatol 2015;67:686-91.

5. Yang L, Wang L, Wang $X$, et al. A possible role of intestinal microbiota in the pathogenesis of ankylosing spondylitis. Int $\mathrm{J} \mathrm{Mol}$ Sci 2016;17:2126.

6. Manasson J, Scher JU. Spondyloarthritis and the microbiome: new insights from an ancient hypothesis. Curr Rheumatol Rep 2015;17:10.
7. De Wilde K, Debusschere K, Beeckman S, et al. Integrating the pathogenesis of spondyloarthritis: gut and joint united? Curr Opin Rheumatol 2015;27:189-96.

8. Eppinga $\mathrm{H}$, Konstantinov SR, Peppelenbosch MP, et al. The microbiome and psoriatic arthritis. Curr Rheumatol Rep 2014;16:407.

9. Coit $P$, Sawalha $A H$. The human microbiome in rheumatic autoimmune diseases: a comprehensive review. Clinical Immunology 2016;170:70-9.

10. Ciccia F, Ferrante A, Guggino G, et al. The role of the gastrointestinal tract in the pathogenesis of rheumatic diseases. Best Pract Res Clin Rheumatol 2016;30:889-900.

11. Tito RY, Cypers H, Joossens M, et al. Brief report: dialister as a microbial marker of disease activity in spondyloarthritis. Arthritis Rheumatol 2017;69:114-21.

12. Eppinga $\mathrm{H}$, Sperna Weiland CJ, Thio HB, et al. Similar depletion of protective faecalibacterium prausnitzii in psoriasis and inflammatory bowel disease, but not in hidradenitis suppurativa. J Crohns Colitis 2016;10:1067-75.

13. Ritchlin CT, Colbert RA, Gladman DD, et al. Psoriatic arthritis. N Engl J Med 2017;376:957-70.

14. Terslev L, Naredo E, lagnocco A, et al. Defining enthesitis in spondyloarthritis by ultrasound: results of a delphi process and of a reliability reading exercise. Arthritis Care Res 2014;66:741-8.

15. Peluso $R$, lervolino $S$, Vitiello $M$, et al. Extra-articular manifestations in psoriatic arthritis patients. Clin Rheumatol 2015;34:745-53.

16. Gladman DD. Psoriatic arthritis. Dermatol Ther 2009;22:40-55

17. Gossec L, Coates LC, de Wit M, Wm D, et al. Management of psoriatic arthritis in 2016: a comparison of EULAR and GRAPPA recommendations. Nat Rev Rheumatol 2016;12:743-50.

18. Kingsley $\mathrm{GH}$, Kowalczyk A, Taylor $\mathrm{H}$, et al. A randomized placebocontrolled trial of methotrexate in psoriatic arthritis. Rheumatology 2012:51:1368-77.

19. Gossec L, Smolen JS, Ramiro S, et al. European League Against Rheumatism (EULAR) recommendations for the management of psoriatic arthritis with pharmacological therapies: 2015 update. Ann Rheum Dis 2016;75:499-510.

20. Asquith M, Rosenbaum JT. The interaction between host genetics and the microbiome in the pathogenesis of spondyloarthropathies. Curr Opin Rheumatol 2016;28:405-12.

21. Benham H, Robinson PC, Baillet AC, et al. Role of genetics in infection-associated arthritis. Best Pract Res Clin Rheumatol 2015;29:213-25.

22. Shamriz $\mathrm{O}$, Mizrahi $\mathrm{H}$, Werbner $\mathrm{M}$, et al. Microbiota at the crossroads of autoimmunity. Autoimmun Rev 2016;15:859-69.

23. Lories RJ, de Vlam K, VK de. Is psoriatic arthritis a result of abnormalities in acquired or innate immunity? Curr Rheumatol Rep 2012;14:375-82.

24. Mortezavi M, Thiele R, Ritchlin C. The joint in psoriatic arthritis. Clin Exp Rheumatol 2015;33(5 Suppl 93):S20-5

25. Acosta Felquer ML, FitzGerald O. Peripheral joint involvement in psoriatic arthritis patients. Clin Exp Rheumatol 2015;33:26-30.

26. Matt $P$, Lindqvist U, Kleinau S. Up-regulation of CD64-expressing monocytes with impaired Fc $\gamma \mathrm{R}$ function reflects disease activity in polyarticular psoriatic arthritis. Scand J Rheumatol 2015;44:464-73.

27. Al-Mossawi MH, Ridley A, Kiedel S, et al. The role of natural killer cells, gamma delta T-cells and other innate immune cells in spondyloarthritis. Curr Opin Rheumatol 2013;25:434-9.

28. Ryan C, Korman NJ, Gelfand JM, et al. Research gaps in psoriasis: Opportunities for future studies. J Am Acad Dermatol 2014;70:146-67.

29. Berthelot J-M, de la Cochetière M-F, Potel G, et al. Evidence supporting a role for dormant bacteria in the pathogenesis of spondylarthritis. Joint Bone Spine 2013;80:135-40.

30. Abdollahi-Roodsaz S, Abramson SB, Scher JU. The metabolic role of the gut microbiota in health and rheumatic disease: mechanisms and interventions. Nat Rev Rheumatol 2016;12:446-55.

31. Honda K, Littman DR. The microbiota in adaptive immune homeostasis and disease. Nature 2016;535:75-84.

32. Ciccia F, Rizzo A, Triolo G. Subclinical gut inflammation in ankylosing spondylitis. Curr Opin Rheumatol 2016;28:89-96.

33. Miossec P, Korn T, Kuchroo VK. Interleukin-17 and Type 17 Helper T Cells. N Engl J Med Overseas Ed 2009;361:888-98.

34. Ciccia F, Guggino G, Rizzo A, et al. Dysbiosis and zonulin upregulation alter gut epithelial and vascular barriers in patients with ankylosing spondylitis. Ann Rheum Dis 2017;76:1123-32.

35. Pianta A, Arvikar SL, Strle K, et al. Two rheumatoid arthritis-specific autoantigens correlate microbial immunity with autoimmune responses in joints. J Clin Invest 2017;127:2946-56.

36. Scher JU, Ubeda C, Artacho A, et al. Decreased bacterial diversity characterizes the altered gut microbiota in patients with psoriatic 
arthritis, resembling dysbiosis in inflammatory bowel disease. Arthritis Rheumatol 2015;67:128-39.

37. Lindqvist $\mathrm{U}$, Kristjánsson G, Pihl-Lundin I, et al. Patients with psoriatic arthritis have an increased number of lymphocytes in the duodenal mucosa in comparison with patients with psoriasis vulgaris. J Rheumatol 2006;33:924-7.

38. Scarpa R, Manguso F, D'Arienzo A, et al. Microscopic inflammatory changes in colon of patients with both active psoriasis and psoriatic arthritis without bowel symptoms. J Rheumatol 2000;27:1241-6.

39. Van Praet L, Van den Bosch F, Mielants H, et al. Mucosal inflammation in spondylarthritides: past, present, and future. Curr Rheumatol Rep 2011:13:409-15.

40. Schatteman L, Mielants H, Veys EM, et al. Gut inflammation in psoriatic arthritis: a prospective ileocolonoscopic study. $J$ Rheumatol 1995;22:680-3.

41. Ciccia F, Guggino G, Ferrante A, et al. Interleukin-9 overexpression and th9 polarization characterize the inflamed gut, the synovial tissue, and the peripheral blood of patients with psoriatic arthritis. Arthritis Rheumatol 2016;68:1922-31.

42. Lin L, Zhang J. Role of intestinal microbiota and metabolites on gut homeostasis and human diseases. BMC Immunol 2017;18:2.

43. Gill T, Asquith M, Rosenbaum JT, et al. The intestinal microbiome in spondyloarthritis. Curr Opin Rheumatol 2015;27:319-25.

44. Kump PK, Krause R, Allerberger F, et al. Faecal microbiota transplantation-the Austrian approach. Clinical Microbiology and Infection 2014;20:1106-11.

45. Cammarota G, Pecere S, laniro G, et al. Principles of DNA-based gut microbiota assessment and therapeutic efficacy of fecal microbiota transplantation in gastrointestinal diseases. Dig Dis 2016;34:279-85.

46. Austin M, Mellow M, Tierney WM. Fecal microbiota transplantation in the treatment of clostridium difficile infections. Am J Med 2014:127:479-83

47. van Nood E, Vrieze A, Nieuwdorp M, et al. Duodenal infusion of donor feces for recurrent Clostridium difficile. N Engl J Med 2013;368:407-15.

48. Cammarota G, Masucci L, laniro G, et al. Randomised clinical trial: faecal microbiota transplantation by colonoscopy vs. vancomycin for the treatment of recurrent Clostridium difficile infection. Aliment Pharmacol Ther 2015;41:835-43.

49. Lee $\mathrm{CH}$, Steiner T, Petrof EO, et al. Frozen vs fresh fecal microbiota transplantation and clinical resolution of diarrhea in patients with recurrent clostridium difficile infection. JAMA 2016;315:142-9.

50. Li YT, Cai HF, Wang ZH, et al. Systematic review with metaanalysis: long-term outcomes of faecal microbiota transplantation for Clostridium difficile infection. Aliment Pharmacol Ther 2016;43:445-57.

51. Paramsothy S, Kamm MA, Kaakoush NO, et al. Multidonor intensive faecal microbiota transplantation for active ulcerative colitis: a randomised placebo-controlled trial. The Lancet 2017:389:1218-28.

52. Cui $\mathrm{B}$, Feng $\mathrm{Q}$, Wang $\mathrm{H}$, et al. Fecal microbiota transplantation through mid-gut for refractory Crohn's disease: safety, feasibility, and efficacy trial results. J Gastroenterol Hepatol 2015;30:51-8.

53. Lund $\mathrm{H}$, Brunnhuber $\mathrm{K}$, Juhl $\mathrm{C}$, et al. Towards evidence based research. BMJ 2016;355:i5440.

54. Ciccia F, Ferrante A, Triolo G. Intestinal dysbiosis and innate immune responses in axial spondyloarthritis. Curr Opin Rheumatol 2016;28:352-8.

55. Bravo-Blas A, Wessel H, Milling S. Microbiota and arthritis: correlations or cause? Curr Opin Rheumatol 2016;28:161-7.

56. Kabeerdoss J, Sandhya P, Danda D. Gut inflammation and microbiome in spondyloarthritis. Rheumatol Int 2016;36:457-68.

57. Costello M-E, Robinson PC, Benham H, et al. The intestinal microbiome in human disease and how it relates to arthritis and spondyloarthritis. Best Pract Res Clin Rheumatol 2015;29:202-12.

58. Bazsó A, Szodoray P, Sütő G, et al. Importance of intestinal microenvironment in development of arthritis. A systematic review. Immunol Res 2015;61:172-6.

59. Taneja V. Arthritis susceptibility and the gut microbiome. FEBS Lett 2014;588:4244-9.

60. Rosenbaum JT, Lin P, Asquith M, et al. Does the microbiome play a causal role in spondyloarthritis? Clin Rheumatol 2014;33:763-7.

61. Jenks K, Stebbings S, Burton J, et al. Probiotic therapy for the treatment of spondyloarthritis: a randomized controlled trial. $J$ Rheumatol 2010;37:2118-25.

62. Aggarwal A, Sarangi AN, Gaur P, et al. Gut microbiome in children with enthesitis-related arthritis in a developing country and the effect of probiotic administration. Clinical \& Experimental Immunology 2017;187:480-9.
63. Berntson L, Hedlund-Treutiger I, Alving K. Anti-inflammatory effect of exclusive enteral nutrition in patients with juvenile idiopathic arthritis. Clin Exp Rheumatol 2016;34:941-5.

64. Hatakka K, Martio J, Korpela M, et al. Effects of probiotic therapy on the activity and activation of mild rheumatoid arthritis - a pilot study. Scand J Rheumatol 2003;32:211-5.

65. Mandel DR, Eichas K, Holmes J. Bacillus coagulans: a viable adjunct therapy for relieving symptoms of rheumatoid arthritis according to a randomized, controlled trial. BMC Complement Altern Med 2010;10:1.

66. Zamani B, Golkar HR, Farshbaf S, et al. Clinical and metabolic response to probiotic supplementation in patients with rheumatoid arthritis: a randomized, double-blind, placebo-controlled trial. Int J Rheum Dis 2016;19:869-79.

67. Pineda ML, Thompson SF, Summers K, et al. A randomized, double-blinded, placebo-controlled pilot study of probiotics in active rheumatoid arthritis. Med Sci Monit 2011;17:CR347-54

68. Alipour B, Homayouni-Rad A, Vaghef-Mehrabany E, et al. Effects of Lactobacillus casei supplementation on disease activity and inflammatory cytokines in rheumatoid arthritis patients: a randomized double-blind clinical trial. Int $J$ Rheum Dis 2014;17:519-27.

69. Schorpion A, Kolasinski SL. Can probiotic supplements improve outcomes in rheumatoid arthritis? Curr Rheumatol Rep 2017;19:73.

70. Taylor W, Gladman D, Helliwell P, et al. Classification criteria for psoriatic arthritis: Development of new criteria from a large international study. Arthritis \& Rheumatism 2006;54:2665-73.

71. Fang $\mathrm{C}$, Zhong $\mathrm{H}$, Lin $\mathrm{Y}$, et al. Assessment of the cPAS-based BGISEQ-500 platform for metagenomic sequencing. Gigascience 2017.

72. Qin J, Li Y, Cai Z, et al. A metagenome-wide association study of gut microbiota in type 2 diabetes. Nature 2012;490:55-60.

73. Nielsen HB, Almeida M, Juncker AS, et al. Identification and assembly of genomes and genetic elements in complex metagenomic samples without using reference genomes. Nat Biotechnol 2014;32:822-8.

74. Li J, Jia H, Cai X, et al. An integrated catalog of reference genes in the human gut microbiome. Nat Biotechnol 2014;32:834-41.

75. Mishra A, Makharia GK. Techniques of functional and motility test: how to perform and interpret intestinal permeability. $J$ Neurogastroenterol Motil 2012;18:443-7.

76. Sequeira IR, Lentle RG, Kruger MC, et al. Standardising the lactulose mannitol test of gut permeability to minimise error and promote comparability. PLoS One 2014;9:e99256.

77. Thorsen H, Hansen TM, McKenna SP, et al. Adaptation into danish of the stanford Health Assessment Questionnaire (HAQ) and the Rheumatoid Arthritis Quality of Life Scale (RAQoL). Scand J Rheumatol 2001;30:103-9.

78. Brodszky V, Péntek M, Bálint PV, et al. Comparison of the Psoriatic Arthritis Quality of Life (PsAQoL) questionnaire, the functional status $(\mathrm{HAQ})$ and utility (EQ-5D) measures in psoriatic arthritis: results from a cross-sectional survey. Scand J Rheumatol 2010;39:303-9.

79. Zachariae, Claus Zachariae, Hans lb R, Zachariae R, et al. Dermatology life quality index: data from danish inpatients and outpatients. Acta Derm Venereol 2000;80:272-6.

80. Fransen $\mathrm{J}$, et al. Performance of response criteria for assessing peripheral arthritis in patients with psoriatic arthritis: analysis of data from randomised controlled trials of two tumour necrosis factor inhibitors. Ann Rheum Dis 2006;65:1373-8.

81. Felson DT, Anderson JJ, Boers M, et al. American college of rheumatology preliminary definition of improvement in rheumatoid arthritis. Arthritis \& Rheumatism 1995;38:727-35.

82. Felson DT, Anderson JJ, Lange MLM, et al. Should imporvement in rheumatoid arthritis clinical trials be defined as fifty percent or seventy percent improvement in core set measures, rather than twenty percent? Arthritis \& Rheumatism 1998;41:1564-70.

83. Faria JR, Aarão AR, Jimenez LM, et al. Inter-rater concordance study of the PASI (Psoriasis Area and Severity Index). An Bras Dermatol 2010;85:625-9.

84. Jensen OK, Callesen J, Nielsen MG, et al. Reproducibility of tender point examination in chronic low back pain patients as measured by intrarater and inter-rater reliability and agreement: a validation study. BMJ Open 2013;3:e002532.

85. Klingberg E, Carlsten $\mathrm{H}$, Hilme $\mathrm{E}$, et al. Calprotectin in ankylosing spondylitis - frequently elevated in feces, but normal in serum. Scand J Gastroenterol 2012;47:435-44.

86. Kelly CR, Kahn S, Kashyap P, et al. Update on fecal microbiota transplantation 2015: indications, methodologies, mechanisms, and outlook. Gastroenterology 2015;149:223-37.

87. Wang S, Xu M, Wang W, et al. Systematic review: adverse events of fecal microbiota transplantation. PLoS One 2016;11:e0161174. 
88. Rossen NG, et al. Fecal microbiota transplantation as novel therapy in gastroenterology: A systematic review. World J Gastroenterol 2015;21:5359-71.

89. Girotra M, Garg S, Anand R, et al. Fecal microbiota transplantation for recurrent clostridium difficile infection in the elderly: long-term outcomes and microbiota changes. Dig Dis Sci 2016;61:3007-15.

90. Gweon T-G, Kim J, Lim C-H, et al. Fecal microbiota transplantation using upper gastrointestinal tract for the treatment of refractory or severe complicated clostridium difficile infection in elderly patients in poor medical condition: the first study in an asian country. Gastroenterol Res Pract 2016;2016:1-6.

91. Agrawal M, Aroniadis OC, Brandt LJ, et al. The long-term efficacy and safety of fecal microbiota transplant for recurrent, severe, and complicated clostridium difficile infection in 146 elderly individuals. J Clin Gastroenterol 2016;50:403-7.

92. Di Bella S, Gouliouris T, Petrosillo N. Fecal microbiota transplantation (FMT) for Clostridium difficile infection: focus on immunocompromised patients. J Infect Chemother 2015;21:230-7.

93. Webb BJ, Brunner A, Ford CD, et al. Fecal microbiota transplantation for recurrent Clostridium difficile infection in hematopoietic stem cell transplant recipients. Transpl Infect Dis 2016;18:628-33.

94. Harris PA, Taylor R, Thielke R, et al. Research electronic data capture (REDCap) - A metadata-driven methodology and workflow process for providing translational research informatics support. $J$ Biomed Inform 2009;42:377-81.

95. Altman DG, Doré CJ. Randomisation and baseline comparisons in clinical trials. The Lancet 1990;335:149-53.

96. Normand S-LT, Sykora K, Li P, et al. Readers guide to critical appraisal of cohort studies: 3 . Analytical strategies to reduce confounding. BMJ 2005;330:1021-3.

97. White IR, Horton NJ, Carpenter J, et al. Strategy for intention to treat analysis in randomised trials with missing outcome data. BMJ 2011;342:d40.

98. Liu R, Hong J, Xu X, et al. Gut microbiome and serum metabolome alterations in obesity and after weight-loss intervention. Nat Med 2017;23:859-68.

99. Pedersen HK, Gudmundsdottir V, Nielsen HB, et al. Human gut microbes impact host serum metabolome and insulin sensitivity. Nature 2016;535:376-81.

100. Thaiss CA, Zmora N, Levy M, et al. The microbiome and innate immunity. Nature 2016;535:65-74.

101. McLean MH, Dieguez D, Miller LM, et al. Does the microbiota play a role in the pathogenesis of autoimmune diseases? Gut 2015;64:332-41.

102. Longman RS, Yang Y, Diehl GE, et al. Microbiota: host interactions in mucosal homeostasis and systemic autoimmunity. Cold Spring Harb Symp Quant Biol 2013;78:193-201.

103. Shreiner AB, Kao JY, Young VB. The gut microbiome in health and in disease. Curr Opin Gastroenterol 2015;31:69-75.

104. Hooper LV, Littman DR, Macpherson AJ. Interactions between the microbiota and the immune system. Science 2012;336:1268-73.

105. Round JL, Mazmanian SK. The gut microbiota shapes intestinal immune responses during health and disease. Nat Rev Immunol 2009;9:313-23.

106. Van de Wiele T, Van Praet JT, Marzorati M, et al. How the microbiota shapes rheumatic diseases. Nat Rev Rheumatol 2016;12:398-411.

107. Buttó LF, Haller D. Dysbiosis in intestinal inflammation: cause or consequence. Int J Med Microbiol 2016;306:302-9.

108. Moayyedi P, Surette MG, Kim PT, et al. Fecal microbiota transplantation induces remission in patients with active ulcerative colitis in a randomized controlled trial. Gastroenterology 2015;149:102-9.

109. Rossen NG, Fuentes S, van der Spek MJ, et al. Findings from a randomized controlled trial of fecal transplantation for patients with ulcerative colitis. Gastroenterology 2015;149:110-8.

110. Smolen JS, Schöls M, Braun J, et al. Treating axial spondyloarthritis and peripheral spondyloarthritis, especially psoriatic arthritis, to target: 2017 update of recommendations by an international task force. Ann Rheum Dis 2018;77:3-17.

111. Zhernakova A, Kurilshikov A, Bonder MJ, et al. Population-based metagenomics analysis reveals markers for gut microbiome composition and diversity. Science 2016;352:565-9.

112. Falony $\mathrm{G}$, Joossens $\mathrm{M}$, Vieira-Silva S, et al. Population-level analysis of gut microbiome variation. Science 2016;352:560-4.

113. Forslund K, Hildebrand F, Nielsen T, et al. Disentangling type 2 diabetes and metformin treatment signatures in the human gut microbiota. Nature 2015;528:262-6.

114. Zhang $X$, Zhang $D$, Jia $H$, et al. The oral and gut microbiomes are perturbed in rheumatoid arthritis and partly normalized after treatment. Nat Med 2015;21:895-905

115. Woodworth $\mathrm{MH}$, Neish EM, Miller NS, et al. Laboratory testing of donors and stool samples for fecal microbiota transplantation for recurrent Clostridium difficile Infection. J Clin Microbiol 2017;55:1002-10.

116. Costello SP, Tucker EC, La Brooy J, Bj L, et al. Establishing a fecal microbiota transplant service for the treatment of clostridium difficile infection. Clin Infect Dis 2016;62:908-14.

117. Cammarota $\mathrm{G}$, laniro $\mathrm{G}$, Tilg $\mathrm{H}$, et al. European consensus conference on faecal microbiota transplantation in clinical practice. Gut 2017;66:569-80.

118. Chu ND, Smith MB, Perrotta AR, et al. Profiling living bacteria informs preparation of fecal microbiota transplantations. PLoS One 2017;12:e0170922.

119. Satokari R, Mattila E, Kainulainen V, et al. Simple faecal preparation and efficacy of frozen inoculum in faecal microbiota transplantation for recurrent Clostridium difficile infection--an observational cohort study. Aliment Pharmacol Ther 2015;41:46-53.

120. Arumugam M, Raes J, Pelletier E, et al. Enterotypes of the human gut microbiome. Nature 2011;473:174-80.

121. Kazerouni A, Wein LM. Exploring the efficacy of pooled stools in fecal microbiota transplantation for microbiota-associated chronic diseases. PLoS One 2017;12:e0163956.

122. Vandeputte D, Falony G, Vieira-Silva S, et al. Stool consistency is strongly associated with gut microbiota richness and composition, enterotypes and bacterial growth rates. Gut 2016;65:57-62.

123. Ley RE. The gene-microbe link. Nature 2015;518:S7

124. Li SS, Zhu A, Benes V, et al. Durable coexistence of donor and recipient strains after fecal microbiota transplantation. Science 2016;352:586-9.

125. Spor A, Koren O, Ley R. Unravelling the effects of the environment and host genotype on the gut microbiome. Nat Rev Microbiol 2011;9:279-90

126. Markle JG, Frank DN, Mortin-Toth S, et al. Sex differences in the gut microbiome drive hormone-dependent regulation of autoimmunity. Science 2013;339:1084-8.

127. Xiao L, Estellé J, Kiilerich P, et al. A reference gene catalogue of the pig gut microbiome. Nat Microbiol 2016:16161.

128. Markle JG, Frank DN, Adeli K, et al. Microbiome manipulation modifies sex-specific risk for autoimmunity. Gut Microbes 2014;5:485-93.

129. Mills S, Shanahan F, Stanton C, et al. Movers and shakers: influence of bacteriophages in shaping the mammalian gut microbiota. Gut Microbes 2013;4:4-16.

130. Pfeiffer JK, Virgin HW. Viral immunity. Transkingdom control of viral infection and immunity in the mammalian intestine. Science 2016;351:aad5872.

131. Underhill DM, Pearlman E. Immune interactions with pathogenic and commensal fungi: a two-way street. Immunity 2015;43:845-58.

132. Castelino M, Eyre S, Moat J, et al. The skin microbiome in psoriatic arthritis: methodology development and pilot data. Lancet 2015;385 Suppl 1(Suppl 1):S27.

133. Scher JU, Joshua V, Artacho A, et al. The lung microbiota in early rheumatoid arthritis and autoimmunity. Microbiome 2016;4:60. 